\title{
Barriers LGBTQI+ People Face in the Research Funding Processes
}

Written by the LGBTQI+ focus group of The Inclusion Group for Equity in Research in STEMM (TIGERS).

For written correspondence, please contact roar@tigerinstemm.org or contact us via @tigerinstemm.

This is version 1 of the document - we appreciate comments (either via twitter or email).

\section{Executive summary:}

We present a range of structural issues and biases which affect access to research funding for those who identify as members of the lesbian, gay, bisexual, trans, queer, intersex and related communities (LGBTQI+), and we particularly focus in on science, technology, engineering, mathematics and medicine ${ }^{1}$ (STEMM) disciplines.

We explore the impact of these barriers for members of these communities, and find:

(1) Within the LGBTQI+ community there is a varied spectrum of experience.

(2) There are specific cultural and structural issues that affect LGBTQI+ people, especially those who identify with a non-binary gender and/or as trans.

(3) There is limited data collected by the research councils in the UK on the experiences, funding applications, and awarded money for LGBTQI+ individuals in STEMM.

In the document, we provide a range of recommendations which include raising visibility of LGBTQI+ individuals, having an equality, diversity, inclusion and accessibility (EDI\&A) champion at a senior level, as well as embedding EDI \& A as central facet of the senior management team.

\footnotetext{
${ }^{1}$ In this document we focus on issues associated with barriers with regards to access to funding. We acknowledge that there are other dimensions, e.g. medics and engagement with patient care.
} 
TIGER in STEM - Research Funding Barriers: LGBTQI+ People

\section{Glossary}

Definitions from Stonewall and other other sources

Cis/Cisgender: Someone whose gender identity is the same as the sex they were assigned at birth. Non-trans is also used by some people

EDI \& A: equality, diversity, inclusion and accessibility

EPSRC: Engineering and Physical Sciences Research Council, the UK's main agency for funding research in engineering and the physical sciences.

Heteronormative: the world view that assumes and promotes heterosexuality as the normal or preferred sexual orientation

LGBTQI+: An inclusive term for those who identify as lesbian, gay, bisexual, trans, queer, intersex and more.

NERC: Natural Environment Research Council, the UK's leading public funder of environmental science.

Non-binary: a spectrum of gender identities that are not exclusively masculine or feminine, that is, identities that are outside the gender binary, that can be fluid and fluctuate between them.

Queer: Queer is a term used by those wanting to reject specific labels of romantic orientation, sexual orientation and/or gender identity. It can also be a way of rejecting the perceived norms of the LGBT community (racism, sizeism, ableism etc). Although some LGBT people view the word as a slur, it was reclaimed in the late 80 s by the queer community who have embraced it.

Trans/Transgender: An umbrella term to describe people whose gender is not the same as, or does not sit comfortably with, the sex they were assigned at birth. Trans people may describe themselves using one or more of a wide variety of terms, including (but not limited to) transgender, transsexual, gender-queer (GQ), gender-fluid, non-binary, gender-variant, trans man, trans woman.

UKRI: UK Research and Innovation brings together the seven research councils, Innovate UK and Research England to support world-leading research and innovation 


\section{Barriers LGBTQI+ People Face in the Research Funding Processes}

Written by the LGBTQI+ focus group of The Inclusion Group for Equity in Research in STEMM (TIGERS). For written correspondence, please contact roar@tigerinstemm.org or contact us via @tigerinstemm.

\section{Introduction}

The aim of this document is to provide an overview of the biases and barriers that affect LGBTQI+ ${ }^{2}$ people in the processes relating to applying for research funding as identified and discussed by the LGBTQI+ focus group of The Inclusion Group for Equity in Research (www.tigerinstemm.org). Despite greater acceptance of LGBTQI+ people in the UK over recent decades, levels of acceptance have plateaued (Curtice et al. 2019). In the workplace, a significant number people still face discrimination and harrassment due to their sexual orientation or gender identity (Bachmann \& Gooch 2018). In the USA, a recent survey showed that LGBT people, regardless of age, consistently reported more negative workplace experiences across a number of STEM professions (Cech and Pham 2016). Heteronormative ${ }^{3}$ assumptions in the workplace can therefore lead LGBTQ+ people to downplay their sexual orientation or gender identity to fit in (Rumens \& Kerfoot, 2009, p. 765 from Yoder \& Matthias 2016). Many STEMM disciplines are still seen as largely the preserve of white, able-bodied, heterosexual, cis-gendered men. This leads to many groups of people including women, LGBTQI+ people, people of non-white ethnicities, and the disabled being under-represented. We note that people may be members of one or more of these groups. The heteronormative environments of these disciplines therefore can be very

\footnotetext{
${ }^{2}$ In this document, we use the phrase "LGBTQI+" to represent the entire community of lesbian, gay, bisexual, transgender, queer, questioning, intersex, asexual, genderqueer, nonbinary and persons with marginalized gender identities or sexual orientations. When we quote other studies, we use the set of acronyms used in that article.

${ }^{3}$ As an idea heteronormativity highlights a world view that promotes heterosexuality, and by proxy the gender binary, as the normal or preferred sexual orientation
} 
TIGER in STEM - Research Funding Barriers: LGBTQI+ People

gendered and ableist, pressuring individuals to conform. In light of this, it is perhaps not surprising that high proportions of LGBTQI+ people consider leaving STEM disciplines (IOP/RAS/RSC 2019).

In the workplace, LGBTQI+ scientists face a spectrum of barriers ranging over a sense of not belonging, isolation, exclusion, and structural obstacles ${ }^{4}$. Some of these challenges and burdens are institutional, some are unintended, and others originate with explicit hostility and prejudice towards LGBTQI+ people.

We emphasise here that the LGBTQI+ community is a diverse one, with varied needs. Those who are trans (broadly defined) face particularly significant challenges. For example, rules around birth certificates and legal identification can be a source of serious trouble for T (trans) people, but not for cisgender LGB people. Institutional policies that apply to same-gender partnerships is critically important for partnered LG (lesbian and gay) people and for many partnered T and B (bisexual) people, but can be less of an issue for heterosexual T persons, or for B people with partners of a different gender. The 2019 IOP/RAS/RSC report also noted a significant proportion of LGBT+ people experienced or observed exclusionary behaviours and practices, particularly those who identify as trans or non-binary (IOP/RAS/RSC 2019).

Many members of the LGBTQI+ community are marginalized in other ways as well (e.g. Matthies et al. 2019; Wanelik et al. 2019), including (but not limited to) their race, gender, socio-economic class, dis/ability status, national origin, and citizenship. These attributes, and their social impact, cannot be separated from an individual's experience as an LGBTQI+ person; LGBTQ+ scientists who are also members of a ethnic minority may feel even more pressure to hide their LGBTQ+ status (Atherton et al. 2016), so as to avoid compounding the exclusion they already face.

In this document, we outline barriers that may limit equitable engagement in STEMM with a specific focus on access to resources and applying for funding. While in isolation these barriers

\footnotetext{
${ }^{4}$ These are obstacles imposed by an institution, either intentionally or unintentionally, that prejudice LGBTQI+ individuals, such as gender entries within forms assuming a cis-binary gender identity.
} 
TIGER in STEM - Research Funding Barriers: LGBTQI+ People

may seem significant (and for many individuals they are insurmountable without structural change or support), we do note that the majority of LGBT+ people broadly felt comfortable in the workplace (IOP/RAS/RSC 2019).

\section{Institutional Barriers}

In STEMM disciplines, obtaining grant income is an essential part of being an independent researcher. Yet competition is tough; grant success rates for the EPSRC ${ }^{5}$ stood at less than $30 \%$ for $2017-2018^{6}$, but was much lower for $\mathrm{NERC}^{7}$, at less than $20 \%^{8}$. As far as we are able to determine, the parent organisation of EPSRC and NERC, UKRI ${ }^{9}$, does not routinely collect data on whether applicants are $\mathrm{LGBTQI}_{+}$, and therefore pinpointing where specific barriers might exist is difficult. Indeed, UKRI recently conducted their own Equality Impact Assessment ${ }^{10}$, where they considered, amongst others, the impact of their Future Leaders Fellowship Scheme (FLFS) on various groups with protected characteristics. For those who are undergoing gender reassignment, consideration has been given to medical absences, and adjustment of UKRI records (including Gateway to Research, and communication materials). For the former, medical absences due to gender reassignment are in principle allowable by all UKRI fellowships, however there are no explicit guidelines for this procedure which poses issues for fellowship holders in terms of disclosure and introduces room for discrimination. For the latter, adjustments would be made under exceptional circumstances at the discretion of the individual research council. While there are positive steps to support trans individuals, such as proposed changes to the wording of UKRI Terms and Conditions, these changes have not been shared publicly and thus we cannot confirm how reasonable or useful they are in practice. If we focus on just sexual orientation, their

\footnotetext{
${ }^{5}$ The Engineering and Physical Sciences Research Council, the UK's main agency for funding research in engineering and the physical sciences.

${ }^{6}$ https://epsrc.ukri.org/funding/fundingdecisions/successrates/

7 The Natural Environment Research Council, the UK's main agency for funding research in environmental science.

${ }^{8}$ https://nerc.ukri.org/funding/application/outcomes/success/

9 UK Research \& Innovation https://www.ukri.org/

10 https://www.ukri.org/files/funding/flf-equality-impact-assessment-pdf/
} 
TIGER in STEM - Research Funding Barriers: LGBTQI+ People

guidance admits that they do not have enough information to assess whether the being LGBQ+ would have a positive or negative impact on FLFS, so no actions are recommended either.

Intervention to address these barriers can occur as 'bottom-up' actions within institutions and also 'top-down' via the funding agencies themselves, and these interventions should be implemented to remedy flaws in our current situation. We have collated here a set of points which we believe act as barriers to LGBTQI+ people applying for funding, which are not faced by straight/nonLGBTQI+ identifying applicants. To support these recommendations, we also present some rationale, and where possible evidence, of issues that may prevent LGBTQI+ people from applying for grants in the first place.

Discrimination and representation: In their recent survey, IOP/RSC/RAS (2019) found that almost $30 \%$ of LGBT+ people have thought about leaving STEM employment due to discrimination or workplace climate. Moreover, almost twice as many LGBT+ people had experienced exclusionary behaviour in the workplace in comparison to non-LGBT+ people, with trans and non-binary people being affected the most (ibid.). Clearly, if the risk of losing LGBTQ+ talent is increased, then this will mean that potentially excellent research ideas are lost even before the funding stage. People who work in stressful environments due, for example, to heteronormative expectations (e.g. on personal presentation, 'normal' with regard family life) or to harassment and exclusion, will also not likely be performing at their best.

However, while visibility and representation matters ${ }^{11}$, especially having LGBTQI+ role models at senior levels in any organisation (Bachmann \& Gooch 2018), there is also the burden of visibility and participation. For example, in the UK, participation in creating submissions for Athena SWAN is biased towards women and people from marginal groups, including those of ethnic minority and who are LGBT (Tzanakou \& Pearce 2019). This places extra pressure on available time to devote to crafting research proposals.

${ }^{11}$ https://www.psychologytoday.com/us/blog/all-things-lgbtg/201711/why-visibility-matters 
As far as we can ascertain, none of the UK's women's fellowships (both charity-based and institutional) have any visible trans-inclusive statements or guidelines under eligibility (and many of them do not consider non-binary individuals). Often, the eligibility of a transgender applicant is left to the interpretation of the assessment panel.

Prizes and awards are a cornerstone for recognition of achievements in academia, that can greatly underpin one's standing, especially with regard to obtaining secure employment, promotion, and further awards (Merton 1968, Merton 1988). Given the discrimination identified above, further work needs to be done to investigate if LGBTQI+ people are recognised for their work, or also experience bias in this regard.

A significant amount of an applicant's track record in STEMM is focussed on their publication output and prior grant history. We have a historic and cultural attitude towards referencing past work with respect to an individual's surname, e.g. through use of citations and the $\mathrm{H}$-index. This is known to be problematic for those who change their name, e.g. through marriage or caste reasons (Holliday et al. (2015), COPE Case 13-02), but may also impact individuals who change their name during their gender transition. Some learned societies have made recommendations to address the need to systematically accommodate name changes in publication records (Atherton et al. 2016).

Networking: Success in STEMM can be dependent on having an international network of collaborators; indeed promotion criteria from leading universities have this as a key promotion criteria. However, for many LGBTQI+ people, travel to international meetings (a common location for networking) can be difficult to impossible ${ }^{12}$.

Reasons that impact members of the LGBTQI+ communities include:

- The legal status of LGBTQI+ persons in the destination or transiting country

\footnotetext{
${ }^{12}$ https://www.fitfortravel.nhs.uk/advice/general-travel-health-advice/lesbian-gay-bisexual-andtransgender-lgbt-travellers
} 
TIGER in STEM - Research Funding Barriers: LGBTQI+ People

- Intersectional issues with LGBTQI+ such as ethnicity, gender, religious beliefs can augment potential negative impact.

- Transgender people may face issues if the gender given on their passport is not the same at the time of travel ${ }^{13}$.

- LGBTQI+ people taking medications (e.g. antiretrovirals or hormones) may face problems if that medication is questioned at customs or not available in other countries.

Networking as an openly LGBTQI+ person in the presence of heteronormative expectations and heterosexism can also be very difficult. For example, at conference dinners and receptions, there is still the expectation for partners to be of the opposite sex. Networking in countries where being LGBTQI+ is problematic can make this even more difficult. While an annual review of the human rights situation with regards LGBTQI perspectives in Europe is available (ILGA, 2018), those countries with a poor track record are visited at an individual's own risk.

These barriers may be difficult to overcome, but there is hope. For instance, one way in which members of the LGBTQI+ community and their allies are trying to make it more comfortable for non-binary and trans individuals is through the active encouragement for all people to consider including their pronouns in email signatures and conference badge stickers. Not only does this clarify communication, it also provides a visable inclusion of people who may identify as nonbinary and assist in reducing instances of misgendering. However, the attempt to solicit their widespread adoption can be seen as intrinsically / problematically "political".

These 'political' issues can be compounded when social media is involved. For instance, social media policies of institutions, societies, and countries may be in conflict. While generalised statements of "do not bring us into disrepute" may protect the organisation, they do not provide active protections for individuals who may be involved in LGBTQI+ activities to promote equality or protect individuals. In terms of networking, this is compounded for conferences that are held

\footnotetext{
${ }^{13}$ https://assets.publishing.service.gov.uk/government/uploads/system/uploads/attachment data/file/2517 03/Applying for a passport additional information.PDF
} 
TIGER in STEM - Research Funding Barriers: LGBTQI+ People

in countries which are actively hostile to LGBTQI+ individuals and still have legislation that, for instance, "limits propaganda aimed at children" and thus social media posts on LGTBQI+ issues can be weaponized to discriminate and prosecute LGBTQI+ individuals.

\section{Direct barriers}

We have identified a number of potential direct barriers to LGBTQI+ people applying for, and being successful in gaining funding. These are detailed below, in no particular order.

- "Demand management" UKRI expects many universities to pre-screen applications before they are submitted for funding, and this is especially the case for universities that are put under "demand management"14. To date few, if any, attempts have been made to look at how LGBTQI+ people may be discriminated against during internal selection.

- LGBTQI+ people are less likely to be asked to interview for potential jobs in the UK (Drydakis 2015), which leaves open the question if LGBTQI+ researchers also face such discrimination.

- Interview panels are likely to discriminate against genderqueer/nonconforming or trans individuals, in the same way that women and people of colour are discriminated against at this stage [We will cross reference to a future document on barriers re: race]. We know of instances where LGBTQI+ applicants have been told to "butch up and use male pronouns please" during Institutional pre-screening for grant applications, or just told to change the way they look in general (Freeman 2018).

- Those at the intersection may face even greater discrimination at all stages of the process, as STEMM diversity and inclusion policies are framed via binary representations of gender, sexuality, religion, race, disability and class, rarely accounting for those at the 'intersection'. Lessons can be learned from arts funders, such as the Jerwood Foundation who have introduced a toolkit to consider these perspectives ${ }^{15}$.

- Schemes which require partnership with countries that discriminate against or criminalise same-sex attracted and/or trans individuals.

\footnotetext{
${ }^{14}$ https://nerc.ukri.org/funding/available/researchgrants/demand/

15 https://jerwoodarts.org/projects/weston-jerwood-creative-bursaries-2017-19/toolkit/
} 
TIGER in STEM - Research Funding Barriers: LGBTQI+ People

- Trans identities are almost impossible to not disclose on an application, owing to name changes, which will be visible through publication history and possibly other identity documents.

- Extended career gaps for trans-specific medical issues; the recovery process is not as straightforward as one expects with most other types of surgeries. A non-negligible number of cases can lead to medium-/long-term disability or complications.

\section{Positive Actions for the future}

Universities, research institutions and learned societies can take several steps to provide positive actions for LGBTQI+ people. For example, taking as a starting point findings from IOP/RAS/RSC (2019):

- Build a visibly welcoming STEMM community

- Review and improve policies particularly with to account for those at the intersection.

- Introduce and improve training

Role models for LGBTQI+ inclusion in science should be encouraged and supported to lead initiatives such as creating a peer-support network for employees, create development opportunities, collaborate with other LGBTQI+ organizations especially in STEMM, with a view to eventually becoming recognised for LGBTQI+ inclusion (Reavey 2019). A network of European universities has recently produced a Manifesto for more inclusive universities, recognising that they need to be more inclusive for all under-represented groups, including LGBTQI+ staff and students (LERU 2019).

TIGERS have the following recommendations:

- Learned Societies establish written best practices and Codes of Conduct that explicitly support LGBTQI+ people in STEMM. 
TIGER in STEM - Research Funding Barriers: LGBTQI+ People

- UKRI engage with Stonewall's best practices in the workplace, eg the UK Workplace Equality Index ${ }^{16}$, and improve training of staff who are responsible for selecting reviewers, grant panel members, and other roles where biases can occur

- grant review panels are monitored by an EDI champion for potential bias and discrimination. We strongly recommend that there is senior leadership engagement, not only via an EDI champion, but recognise that EDI is a critical facet for sustainable management decisions within the University environment.

- UKRI / Research Councils employ an EDI champion or advisor who observes directly research-funding interviews, and who can be the first point of contact for any dispute resolution process regarding perceived unfair treatment at interview.

- workplaces promote LGBTQI+ inclusive practices and mentoring programmes.

- $\quad$ any $L G B T Q I+$ harassment be treated as scientific misconduct.

- processes are reviewed to ensure that there are a diverse range of individuals involved in making decisions, and that LGBTQI+ individuals, and those that have experience of multiple challenges are actively included.

\section{Sources}

Atherton, T.J., Barthelemy, R.S., Deconinck, W., Falk, M.L., Garmon, S., Long, E., Plisch, M., Simmons, E.H., Reeves, K. (2016) LGBT climate in physics: Building an inclusive community. American Physical Society.

Bachmann, C.L., Gooch, B. (2018) LGBT In Britain: Stonewall Work Report. Stonewall, London. $17 \mathrm{pp}$

Cech, E.A., Pham, M.V. (2017) Queer in STEM organizations: workplace disadvantages for LGBT employees in STEM related federal agencies. Social Sciences 6, 12 pp. doi:10.3390/socsci6010012

COPE Case 13-02 Change in author's name after publication https://publicationethics.org/case/change-author\%E2\%80\%99s-name-after-publication

Drydakis, N. (2015) Sexual orientation discrimination in the United Kingdom's labour market: A field experiment. Human Relations 68, 1769-1796. DOI: 10.1177/0018726715569855

${ }^{16}$ https://www.stonewall.org.uk/creating-inclusive-workplaces/workplace-equality-indices/uk-workplaceequality-index 
TIGER in STEM - Research Funding Barriers: LGBTQI+ People

Curtice, J., Clery, E., Perry, J., Phillips M. Rahim, N. (eds.) (2019) British Social Attitudes: The 36th Report. London: The National Centre for Social Research

English, R. Fenby-Hulse, K. (2019) Documenting diversity: the experiences of LGBTQ+ doctoral researchers in the UK. International Journal of Doctoral Studies 14, 403-430.

doi.org/10.28945/4328

Freeman, J. (2018) LGBTQ scientists are still left out. Nature 559, 27-28. doi: 10.1038/d41586$\underline{018-05587-y}$

Holliday, E.B., Jagsi, R., Wilson, L., Choi, M., Thomas, C.R., and Fuller, C.D. (2015) Gender Differences in Publication Productivity, Academic Position, Career Duration and Funding Among U.S. Academic Radiation Oncology Faculty. Academic Medicine 91, 1158-1163 doi: 10.1097/ACM.0000000000000229

ILGA (2018) Europe: Annual Review of the Human Rights Situation of Lesbian, Gay, Bisexual, Trans and Intersex People in Europe https://www.ilgaeurope.org/sites/default/files/2018/full annual review.pdf

IOP/RSC/RAS (2019) Exploring the workplace for LGBT+ physical scientists. A report by the Institute of Physics, Royal Astronomical Society and Royal Society of Chemistry. https://www.rsc.org/campaigning-outreach/campaigning/incldiv/lgbt-report/

LERU (2019) Equality, diversity and inclusion at universities: the power of a systematic approach. LERU position paper https://www.leru.org/publications/equality-diversity-andinclusion-at-universities

Mattheis, A., Cruz-Ramírez De Arellano, D., Yoder, J.B. (2019) A Model of Queer STEM Identity in the Workplace. Journal of Homosexuality doi: 10.1080/00918369.2019.1610632

Merton, R.K. (1968) The Matthew Effect in Science. Science 159, 56-63. doi: 10.1126/science. 159.3810 .56

Merton, R.K. (1988) The Matthew Effect in Science, II: cumulative advantage and the symbolism of Intellectual Property. Isis 79, 606-623 doi: 10.1086/354848

Reavey, N. (2019) Stepping up to be a role model for LGBTQ inclusion in science. Career Column. Nature https://www.nature.com/articles/d41586-019-01146-1

Stout, J.G., Wright, H.M. (2016) Lesbian, Gay, Bisexual, Transgender, and Queer Students' Sense of Belonging in Computing: An Intersectional Approach. Computing in Science \& Engineering 18, 24-30. https://doi.org/10.1109/MCSE.2016.45 
TIGER in STEM - Research Funding Barriers: LGBTQI+ People

Tzanakou, C., Pearce, R. (2019) Moderate feminism within or against the neoliberal university? The example of Athena SWAN. Gender, Work \& Organization 26, 1191-1211 doi:

$\underline{10.1111 / \mathrm{gwao} .12336}$

Wanelik, K.M., Griffin, J.S., Head, M., Ingleby, F.C., Lewis, Z. (2019) Breaking barriers: The effect of protected characteristics and their intersectionality on career transition in academics. BioRxiv, p.593905. doi: https://doi.org/10.1101/593905

Yoder, J.B., Mattheis, A. (2016) Queer in STEM: Workplace Experiences Reported in a National Survey of LGBTQA Individuals in Science, Technology, Engineering, and Mathematics Careers, Journal of Homosexuality 63, 1-27. doi: 10.1080/00918369.2015.1078632 